\title{
Estimation of One-parameter Exponential Family Under Entropy Loss Function Based on Record Values ${ }^{1}$
}

\author{
Jinping $\mathrm{Li}^{\mathrm{a}}$, Haiping Ren ${ }^{\mathrm{b}}$ \\ College of Information Science and Technology, Hainan University, Haikou,570228, China \\ School of Software, Jiang Xi University of Science and Technology, Nanchang,330013, China
}

\begin{abstract}
In this paper, we consider one-parameter exponential family and obtain the minimum variance unbiased estimator, Bayes and empirical Bayes estimators of the unknown parameter based on record values under entropy loss function. The admissibility and inadmissibility of a class of inverse linear estimators are also discussed based on upper records.
\end{abstract}

Index Terms: admissibility;Bayes and empirical Bayes estimators; record values; entropy loss function

(C) 2012 Published by MECS Publisher. Selection and/or peer review under responsibility of the Research Association of Modern Education and Computer Science.

\section{Introduction}

Record values and the associated statistics are of interest and importance in the areas of meteorology, sports and economics .Chandler (1952)laucnched a statistical study of the record values, record times and inter record times. Many authors have studied record values and the associated statistics; see, for example, Nagaraja(1988), Ahsanullah (1990) and Arnold et al. $(1992,1998)$. There are some papers on estimation and prediction for parameters of some life distributions based on records. See for example Ahmed and Soliman (2008), and Jaheen (2004) ,Ahmadi and Doostparast (2006), Asgharzadeh(2009) and references therein.

Let $X_{1}, X_{2}, \cdots$ be a sequence of independent and identically distributed (iid) random variables with cumulative distribution function (cdf) $F(x)$ and probability density function (pdf) $f(x)$. For $n \geq 1$, define

\footnotetext{
${ }^{1}$ This work is partially supported by Natural Science Foundation of Jiangxi Province of China,NO. 2010GZS0190;Foundation of Jiang'xi Educational Committee, NO.GJJ11600,GJJ11479

* Corresponding author.

E-mail address: 479456011@qq.com; chinarhp@163.com
} 


$$
U(1)=1, U(n+1)=\min \left\{j: j>U(n), X_{j}>X_{U(n)}\right\}
$$

The sequence $\left\{X_{U(n)}\right\}$ is known as upper record values.

This paper will discuss the Estimation of parameter from a special one-parameter exponential family,and the admissibility of a class of inverse linear estimators of are also studied based on upper records.

Let $X_{1}, X_{2}, \cdots$ be a sequence of iid random variables from the class $\mathrm{C}$ of one-parameter exponential family with cdf

$$
F(x ; \theta)=1-\exp \{-a(\theta) T(x)\}
$$

and pdf

$$
f(x ; \theta)=a(\theta) T^{\prime}(x) \exp \{-a(\theta) T(x)\}, x>0
$$

where $\theta>0$ and $a(\theta), T(x)$ is a real positive function.

The family in $\mathrm{C}$ is well-known in the lifetime experiments which includes several well-known lifetime distributions such as: Exponential, Pareto, Lomax, Burr type XII, Weibull (one parameter) among others.

\section{Estimation}

In this section,let $X_{U(1)}=x_{1}, \cdots, X_{U(n)}=x_{n}$ be the observed $\mathrm{n}$ upper record values from distribution cdf (1) and pdf (2). Then, the joint distribution of $X_{U(1)}, \cdots, X_{U(n)}$ is given (see Arnold et al. (1998)) by

$$
\begin{aligned}
& f(x ; \theta)=f\left(x_{n} ; \theta\right) \prod_{i=1}^{n-1} H\left(x_{i} ; \theta\right) \\
& =a^{n}(\theta) \exp \left\{-a(\theta) T\left(x_{n}\right)\right\} \prod_{i=1}^{n} T^{\prime}\left(x_{i}\right), 0<x_{1}<\cdots<x_{n}
\end{aligned}
$$

and the marginal pdf of $X_{U(n)}$ is given by

$$
\begin{aligned}
& f_{n}\left(x_{n} ; \theta\right)=f\left(x_{n} ; \theta\right) \frac{R^{n-1}\left(x_{n} ; \theta\right)}{(n-1) !} \\
& =\frac{a^{n}(\theta)}{(n-1) !} T^{n-1}\left(x_{n}\right) T^{\prime}\left(x_{n}\right) \exp \left\{-a(\theta) T\left(x_{n}\right)\right\}
\end{aligned}
$$

where $x=\left(x_{1}, x_{2}, \cdots, x_{n}\right), H(\cdot)$ is the hazard function corresponding to pdf $f(\cdot), H\left(x_{i} ; \theta\right)=\frac{f\left(x_{i} ; \theta\right)}{1-F\left(x_{i} ; \theta\right)}$ 
and $R(x ; \theta)=-\ln (1-F(x ; \theta))$.

Lemma 2.1 Suppose we observe $\mathrm{n}$ upper record values $X_{U(1)}=x_{1}, X_{U(2)}=x_{2}, \cdots, X_{U(n)}=x_{n}$ from distribution cdf (1) and pdf (2).

Then 1) $X_{U(n)}$ is a sufficient statistics for the first $\mathrm{n}$ upper record values.

2) $T\left(X_{U(n)}\right) \sim \Gamma(n, a(\theta))$

Lemma 2.2 Suppose we observe $\mathrm{n}$ upper record values $X_{U(1)}=x_{1}, X_{U(2)}=x_{2}, \cdots, X_{U(n)}=x_{n}$ from distribution cdf (1) and pdf (2) and $a(\theta)=\theta$, Then

1)the maximum likelihood estimator (MLE) of $\theta$ is $\hat{\theta}_{M L E}=n / T$

2) $E\left(\hat{\theta}_{M L E}\right)=\frac{n}{n-1} \theta$

Remark 2.1. Using lemma2.2, we have $E\left[\frac{n-1}{T}\right]=E\left[\frac{n-1}{n} \hat{\theta}_{M L E}\right]=\theta$,thus $\frac{n-1}{T}$ is an unbiased estimator of $\theta$, and is also a function the complete and sufficient statistics $T$.Therefore, by using theorem of Lehmann and Scheff'e(1950), the estimator $\frac{n-1}{T}$ is the minimum variance unbiased estimator(UMVU estimator)of $\theta$, note it by

$$
\hat{\theta}_{U M V U}=\frac{n-1}{T}
$$

In the following discussion, we always suppose $X_{1}, X_{2}, \cdots$ be a sequence of iid random variables from the class $\mathrm{C}$ and $a(\theta)=\theta$. And $\mathrm{n}$ upper record values $X_{U(1)}=x_{1}, X_{U(2)}=x_{2}, \cdots, X_{U(n)}=x_{n}$ are also given. we will consider the Bayesian and empirical Bayesian estimation of $\theta$ under entropy loss function

$$
L(\hat{\theta}, \theta)=\frac{\delta}{\theta}-\ln \frac{\delta}{\theta}-1
$$

Lemma2.3 Let $X_{1}, X_{2}, \cdots, X_{n}$ be a random sample drawn from the probability density $f(x ; \theta)$, under entropy loss function (5), the unique Bayes estimator of $\theta$, say $\hat{\theta}_{B}$, under the asymmetric precautionary loss (2) is given by

$$
\hat{\theta}_{B}=\left[E\left(\theta^{-1} \mid X\right]^{-1}\right.
$$

where $X=\left(X_{1}, X_{2}, \cdots, X_{n}\right)$

Proof. Since the loss in (2.3) is strictly convex, the unique Bayes is obtained from the relation 


$$
\frac{d E(L(\hat{\theta}, \theta) \mid X)}{d \hat{\theta}}=0
$$

which reduces to $(2.4)$.

\subsection{Bayes Estimation}

Suppose $\theta$ has the conjugate family of prior Gamma distributions, $\Gamma(\alpha, \beta)$, with density

$$
\pi(\theta \mid \alpha, \beta)=\frac{\beta^{\alpha}}{\Gamma(\alpha)} \theta^{\alpha-1} e^{-\beta \theta}, \theta>0
$$

where $\alpha>0$ and $\beta>0$. Note that the limiting case $\alpha, \beta \rightarrow 0$ gives the usual noninformative prior for $\pi(\theta) \propto \theta^{-1}$ (see Berger (1985)).

It is easy to verify that the posterior distribution of $\theta$ is $\Gamma\left(n+\alpha, T\left(x_{n}\right)+\beta\right)$, and from lemma 2.3 the unique Bayes estimatorof $\theta$, say $\hat{\theta}_{B}$, is given by

$$
\hat{\theta}_{B}=\left[E\left(\theta^{-1} \mid X\right)\right]^{-1}=\left(\frac{T+\beta}{n+\alpha-1}\right)^{-1},
$$

where $T \equiv T\left(X_{U(n)}\right)$

Thus

$$
\hat{\theta}_{B}=\left(\frac{1}{n+\alpha-1} T+\frac{\beta}{n+\alpha-1}\right)^{-1},
$$

which is of the form $(c T+d)^{-1}$

Remark 2.2. For the noninformative prior $\pi(\theta) \propto \theta^{-1}$, the posterior distribution of $\theta$ is $\Gamma\left(n, T\left(x_{n}\right)\right)$ and we obtain the generalized Bayes estimator

$$
\hat{\theta}_{*}=\frac{T\left(X_{U(n)}\right)}{n-1}
$$

\subsection{Empirical Bayes Estimation}

Assume that the conjugate family of prior distributions for $\theta$ is the family of gamma distributions, $\Gamma(\alpha, \beta)$ with parameter $\alpha$ and $\beta$,and prior parameter $\beta$ is unknown. we may use the empirical Bayes approach 
to get its estimate. From (3) and (7), we calculate the marginal pdf of $X=\left(X_{U(1)}, \cdots, X_{U(n)}\right)$, with density

$$
\begin{aligned}
& m(x \mid \beta)=\int_{0}^{\infty} f(x \mid \theta) \pi(\theta \mid \beta) d \theta \\
& =\int_{0}^{\infty} \theta^{n} \exp \left\{-\theta T\left(x_{n}\right)\right\} \prod_{i=1}^{n} T^{\prime}\left(x_{i}\right) \frac{\beta^{\alpha}}{\Gamma(\alpha)} \theta^{\alpha-1} e^{-\beta \theta} d \theta \\
& =\frac{\beta^{\alpha}}{\Gamma(\alpha)} \frac{\Gamma(n+\alpha)}{\left(\beta+T\left(x_{n}\right)\right)^{n+\alpha}} \prod_{i=1}^{n} T^{\prime}\left(x_{i}\right)
\end{aligned}
$$

Based on $m(x \mid \beta)$, we obtain an estimate $\hat{\beta}$ of $\beta$. The MLE of $\beta$ is $\hat{\beta}=\frac{\alpha}{n} T\left(X_{U(n)}\right)$

Now, by substituting $\hat{\beta}$ for $\beta$ in the Bayes estimator, we obtain the empirical Bayes estimator as

$$
\hat{\theta}_{E B}=\left(\frac{1}{n+\alpha-1} T+\frac{1}{n+\alpha-1} \frac{\alpha}{n} T\right)^{-1}=\left[\frac{n+\alpha}{n(n+\alpha-1)} T\right]^{-1}
$$

Admissibility of $(c T+d)^{-1}$

Note that the estimators obtained in Section 2 are special cases of the more general class of inverse linear estimators of the form $(c T+d)^{-1}$. In this section we always let $c^{*}=\frac{1}{n-1}, T=T\left(X_{U(n)}\right)$

In the rest of this paper, these estimators are compared on the basis of their risks under the loss (2.3). We also obtain conditions under which linear estimators are admissible in terms of risk.

Theorem 3.1 The estimator $(c T+d)^{-1}$ is admissible, provided $0 \leq c<c^{*}$ and $d>0$.

Proof. From (2.5), when $\alpha>0$ and $\beta>0$, the coefficient of $T\left(X_{U(n)}\right)$ is strictly between 0 and $c^{*}$, and the constant $\frac{\beta}{n+\alpha-1}$ is strictly bigger than 0 . This proves that $(c T+d)^{-1}$ is admissible for $0<c<c^{*}$ and $d>0$. When $c=0, d>0$, the estimator $(c T+d)^{-1}$ is admissible since it is the only estimator for which $R(\theta, d)=0$ when $\theta=d$.

Theorem 3.2 The estimator $(c T+d)^{-1}$ is admissible, provided $c=c^{*}$ and $d \geq 0$.

Proof. The case $c=c^{*}$ and $d>0$ is considered first.Let $\theta$ and consider the prior $\pi_{k}(\theta)$ with density

$$
\pi_{k}(\theta)=\frac{\beta^{1 / k}}{\Gamma(1 / \mathrm{k})} \theta^{1 / k-1} e^{-\beta \theta}, \beta>0, k>0
$$


If $\mathrm{A}$ is a nondegenerate convex subset of $(0,+\infty)$, it can be shown that there exists a $k_{0}$ such that $\int_{A} \pi_{k}(\lambda) d \lambda \geq \varepsilon$ for some $\varepsilon>0$ and all $k \geq k_{0}$.

In fact,

$$
\int_{A} \beta^{\frac{1}{\bar{k}}} \theta^{\frac{1}{k}-1} e^{-\beta \theta} d \theta=\int_{A \cap(0,1)} \beta^{\frac{1}{k}} \theta^{\frac{1}{k}-1} e^{-\beta \theta} d \theta+\int_{A \cap(1,+\infty)} \beta^{\frac{1}{k}} \theta^{\frac{1}{k}-1} e^{-\beta \theta} d \theta
$$

Because $\beta^{\frac{1}{k}} \rightarrow 1 \quad(k \rightarrow \infty)$

So the form equation $\geq \frac{1}{2}\left[\int_{A \cap(0,1)} \theta^{\frac{1}{k}-1} \cdot e^{-\beta \theta} d \theta+\int_{A \cap[1,+\infty)} \theta^{\frac{1}{k}-1} \cdot e^{-\beta \theta} d \theta\right],\left(k>k_{0}\right)$

Let $\varepsilon_{0}=\frac{1}{2}\left[\int_{A \cap(0,1)} \theta^{\frac{1}{k}-1} \cdot e^{-\beta \theta} d \theta+\int_{A \cap[1,+\infty)} \theta^{\frac{1}{k}-1} \cdot e^{-\beta \theta} d \theta\right]$,

then the conclusion have proved.

The formal Bayes estimator with respect to $\pi_{k}(\theta)$ under the loss (5) can be derived as in (8) which is given as

$$
\hat{\theta}_{k}=\left(\frac{T+\beta}{n+1 / k-1}\right)^{-1}
$$

Let $\hat{\theta}(X)=\left(\frac{T+\beta}{n-1}\right)^{-1}, F(\theta, t)$ is the jiont probability density function of $(\theta, T)$.

Then $d F(\theta, t)=\frac{\beta^{\frac{1}{k}}}{\Gamma(1 / \mathrm{k})} \theta^{1 / k-1} e^{-\beta \theta} \cdot \frac{\theta^{n}}{\Gamma(n)} t^{n-1} e^{-\theta t} d \theta d t$

The difference of the Bayes risks with respect to $\hat{\theta}_{k}$ and $\hat{\theta}$ is

$$
\begin{aligned}
& r\left(\pi_{k}, \hat{\theta}\right)-r\left(\pi_{k}, \hat{\theta}_{k}\right)=E L(\hat{\theta}, \theta)-E L\left(\hat{\theta}_{k}, \theta\right) \\
& =\int_{0}^{+\infty} \int_{0}^{+\infty}\left(\frac{\hat{\theta}}{\theta}-\ln \frac{\hat{\theta}}{\theta}-1\right)-\left(\frac{\hat{\theta}_{k}}{\theta}-\ln \frac{\hat{\theta}_{k}}{\theta}-1\right) d F(\theta, t) \\
& =p(k) \int_{0}^{+\infty} \int_{0}^{+\infty} \frac{1}{\theta(T+\beta)} d F(\theta, t)-q(k)
\end{aligned}
$$

where $p(k)=\frac{-1}{k}, q(k)=\ln \frac{n-1}{n+1 / k-1}$ and $p(k) \rightarrow 0 \quad q(k) \rightarrow 0, k \rightarrow+\infty$

For any prior distribution $\Gamma(a, b)$ of $\theta$,the following conclusion is always tenable 


$$
\begin{aligned}
& E\left[\frac{1}{\theta(T+\beta)}\right] \leq E\left[\frac{1}{\theta T}\right] \\
& =\int_{0}^{+\infty} \int_{0}^{+\infty} \frac{1}{\theta t} \cdot \frac{t^{n-1}}{\Gamma(n)} \theta^{n} e^{-\theta t} \cdot \frac{\beta^{\alpha}}{\Gamma(\alpha)} \theta^{\alpha-1} e^{-\beta \theta} d \theta d t \\
& =\int_{0}^{+\infty}\left[\int_{0}^{+\infty} t^{(n-1)-1} e^{-\theta t} d t\right] \cdot \frac{\beta^{\alpha}}{\Gamma(\alpha) \Gamma(\mathrm{n})} \theta^{(n+\alpha-1)-1} e^{-\beta \theta} d \theta \\
& =\int_{0}^{+\infty} \frac{\Gamma(n-1)}{\theta^{n-1}} \cdot \frac{\beta^{\alpha}}{\Gamma(\alpha) \Gamma(\mathrm{n})} \theta^{(n+\alpha-1)-1} e^{-\beta \theta} d \theta \\
& =\frac{\Gamma(n-1) \cdot \beta^{\alpha}}{\Gamma(\alpha) \Gamma(\mathrm{n})} \cdot \frac{\Gamma(\alpha)}{\beta^{\alpha}}=\frac{1}{n-1}<+\infty
\end{aligned}
$$

Thus, $\lim _{k \rightarrow \infty}\left(r\left(\pi_{k}, \hat{\theta}\right)-r\left(\pi_{k}, \hat{\theta}_{k}\right)\right)=0$,

Therefore, $(c T+d)^{-1}$ is admissible for any $\beta>0$ by Blyth's lemma. This proves that $(c T+d)^{-1}$ is admissible for $c=c^{*}$ and $d>0$.

For the case $c=c^{*}$ and $d=0$, it is seen that $\left(c^{*} T\right)^{-1}$ is the limit of Bayes estimator relative to gamma prior $\Gamma(a, b)$, as $\alpha, \beta \rightarrow 0$. To prove the admissibility of $\left(c^{*} T\right)^{-1}$, it is easy to verify that the Bayes risk difference $r\left(\pi_{k},\left(c^{*} T\right)^{-1}\right)-r\left(\pi_{k}, \hat{\theta}_{B}\right)$ converges to zero as $\alpha, \beta \rightarrow 0$, which guarantees the admissibility of $\left(c^{*} T\right)^{-1}$.

Theorem 3.3 Let the parameter space be $(0,+\infty)$ and the action space be $[0,+\infty)$ The linear estimator $(c T+d)^{-1}$ is inadmissible under the loss function (2.3) whenever one of the following conditions holds:

(i) $c<0$ or $d<0$;

(ii) $0<c \neq c^{*}, d=0$

(iii) $c>c^{*}$ and $d \geq 0$

Proof. To see (i), note that $(c T+d)^{-1}$ takes on negative values with positive probability. Therefore $(c T+d)^{-1}$ is dominated by $\max \left(0,(c T+d)^{-1}\right)$ in this case.

For the case (ii), using lemma 2.1, the risk function of $(c T)^{-1}$ is 


$$
\begin{aligned}
R\left(\theta,(c T)^{-1}\right) & =E\left[\frac{1}{\theta(c T)}-\ln \frac{1}{\theta(c T)}-1\right] \\
& =\frac{1}{c \theta} E \frac{1}{T}+E(\ln T)+\ln \theta+\ln c-1
\end{aligned}
$$

Using lemma2.1, $E \frac{1}{T}=\frac{\theta}{n-1}, E(\ln T)=\psi(n)-\ln \theta$, where $\psi(n)=\frac{\Gamma^{\prime}(n)}{\Gamma(n)}$.

Then derivative of the risk with respect to $\mathrm{c}$ is :

$$
\frac{\partial}{\partial c} R\left(\theta,(c T)^{-1}\right)=-\frac{1}{c^{2}} \frac{1}{n-1}+\frac{1}{c}=\frac{1}{c}\left(1-\frac{1 / c}{n-1}\right)
$$

Then $\frac{\partial}{\partial c} R\left(\theta,(c T)^{-1}\right)<0$ where $0<c<c^{*}=\frac{1}{n-1}$, and $\frac{\partial}{\partial c} R\left(\theta,(c T)^{-1}\right)>0$, where $c>c^{*}$. Thus the risk of $(c T)^{-1}$ is minimized at $c=c^{*}$. Hence the estimator $(c T)^{-1}$ is dominated by $\left(c^{*} T\right)^{-1}$ in this case.

For case (iii), let us compute the risk function of the inverse linear estimator $(c T+d)^{-1}$.

$$
\begin{aligned}
& R(\theta, c T+d)-R\left(\theta^{*}, c^{*} T+\frac{c^{*}}{c} d\right) \\
& =E\left[\frac{1}{\theta(c T+d)}-\ln \frac{1}{\theta(c T+d)}-\frac{1}{\theta\left(c^{*} T+\frac{c^{*}}{c} d\right)}+\frac{1}{\theta\left(c^{*} T+\frac{c^{*}}{c} d\right)}\right]
\end{aligned}
$$

in the condition (iii), $1-\frac{c}{c^{*}}<0$,so

$$
\begin{aligned}
& R(\theta, c T+d)-R\left(\theta^{*}, c^{*} T+\frac{c^{*}}{c} d\right)=\left\{E\left[\frac{1}{\theta(c T+d)}\left(1-\frac{c}{c^{*}}\right)+\ln \frac{c}{c^{*}}\right]\right\} \\
& \left.\geq\left\{\left(1-\frac{c}{c^{*}}\right) E\left[\frac{1}{\theta c T}\right]+\ln \frac{c}{c^{*}}\right]\right\}=n\left(\frac{c^{*}}{c}-\ln \frac{c^{*}}{c}-1\right)>0
\end{aligned}
$$

Therefore, $R\left(\theta,(c T+d)^{-1}\right)$ is minimized at $c=c^{*}$. Hence $(c T+d)^{-1}$ is dominated by $\left(c^{*} T+d\right)^{-1}$ in this case.

Remark 3.1. The estimators $\hat{\theta}_{M L E}$ and $\hat{\theta}_{E B}$ are inadmissible by using theorem 3.3 . They are dominated by the generalized Bayes estimator $\hat{\theta}_{*}=\left(c^{*} T\right)^{-1}$. 


\section{Conclusions}

Based on record statistics, this paper considers the estimation of the unknown parameter from the one parameter exponential family. The maximum likelihood estimator (MLE), Bayes and empirical Bayes estimators are obtained. These estimators all belong to the class of inverse linear estimators of the form $(c T+d)^{-1}$. So, the admissibility and inadmissibility of $(c T+d)^{-1}$ are studied. As a result, the minimum variance unbiased estimator ,but the empirical Bayes estimator and the maximum likelihood estimator are inadmissible

\section{References}

[1] Chandler, K. N. The distribution and frequency of record values. Journal of the Royal Statistical Society. Series B, Vol.14, pp.220-228,1952

[2] Nagaraja, H. N. Record values and related statistics - A review.Communications in Statistics: Theory and Methods, Vol.17,pp. 2223-2238,1988

[3] Ahsanullah, M. Estimation of the parameters of the Gumbel distribution based on the $m$ record values. Computational Statistics and Quarterly, Vol.3,pp.231-239,1990

[4] Arnold, B. C., Balakrishnan, N. and Nagaraja, H. N. A first course in order statistics. John Wiley \& Sons, New York, 1992

[5] Arnold, B. C., Balakrishnan, N., Nagaraja, H. N.Records. New York: John Wiley \& Sons, 1998

[6] Jaheen, Z. F. Empirical Bayes analysis of record statistics based on LINEX and quadratic loss functions.

Computers and Mathematics with Applications, Vol.47, pp.947-954,2004

[7] Ahmed A,Soliman F. M.Bayesian inference using record values from Rayleigh model with application. European Journal of Operational Research. Vol.185,no.2,pp.659-672,2008

[8] Ahmadi J,Doostparast M. Bayesian estimation and prediction for some life distributions based on record Values. Stat Pap. Vol.47,pp.373-392,2006

[9] Jaheen, Z.F.Empirical Bayes Inference for Generalized Exponential Distribution Based on Records,Communications in Statistics - Theory and Methods, Vol.33,no.8,pp. 1851-1861,2004

[10]A. Asgharzadeh.On Bayesian estimation from exponential distribution based on records.The Korean Statistical Society, Vol.38,no.2,pp. 125-130,2009

[11]Parsian A , Nematollahi N. Estimation of scale parameter under entropy loss function. J of Statistical Planning and Inference, Vol.52,pp.77-91,1996

[12] Lehmann,E.L.,Scheff'e,H.Completeness,similar regions and unbiased estimates.Sankhya Vol.10,pp.305340,1950 